\title{
Medo de cair e a capacidade funcional de idosos a viver na comunidade Fear of falling and physical fitness in community-dwelling older adults Miedo a caer y capacidad funcional de mayores a vivir en la comunidad Vania Brandao de Loureiro, Margarida Isabel Boteta Gomes, Ana Ruivo Alves Instituto Politécnico de Beja (Portugal)
}

\begin{abstract}
Resumo. O medo de cair é apontado como consequência psicológica proveniente da perda de funcionalidade e equilíbrio inerentes ao envelhecimento. Manter bons níveis de aptidão física poderá ser a chave para preservar a qualidade de vida e independência dos idosos. Este estudo tem como objetivo verificar qual a relação do medo de cair com a aptidão física de idosos inseridos em programas de exercício físico. A amostra contemplou 308 idosos, com idade > a 65 anos (86.8\% do sexo feminino; idade $74.78 \pm 5.72$ anos, altura 1.56 $\pm 0.08 \mathrm{~m}$, peso $67.13 \pm 12.14 \mathrm{~kg}$ ), do Baixo Alentejo, Portugal. Avaliaram-se as características sociodemográficas, histórico de quedas, medo de cair (FES-I Portugal); nível de atividade física (IPAQ-E - versão curta) e indicadores de aptidão física funcional (Protocolo de Rikli \& Jones, 2013). Verificaram-se associações significativas entre o medo de cair e as variáveis de aptidão física. Baixo medo de cair parece estar relacionado com melhor força dos membros inferiores $(p<.001)$, agilidade e equilíbrio dinâmico $(p<.001)$ e aptidão cardiorrespiratória $(p<.001)$. Os resultados revelam que um baixo medo de cair está relacionado melhor capacidade funcional. No sentido de contribuir para a manutenção e desenvolvimento de capacidades físicas essenciais e evitar uma diminuição de qualidade de vida, reforçamos a importância de programas de exercício físico que incluam o treino de força, agilidade, equilíbrio e aptidão cardiorrespiratória, integrados em intervenções multifatoriais. Estas estratégias poderão, através da promoção e manutenção das capacidades funcionais, criar uma maior perceção de competência e consequentemente reduzirem o medo de cair.
\end{abstract}

Palavras chave: Força, Envelhecimento saudável, Quedas, Exercício, Promoção da Saúde.

Abstact. The fear of falling is pointed out as a psychological consequence resulting from the loss of functionality and balance inherent to aging. Maintaining good levels of physical fitness may be the key to preserve the quality of life and independence of the older adult. This study aims to verify the relationship of fear of falling with the physical fitness of older adults inserted in a community physical exercise program. The sample included 308 older adults, aged $>65$ years $(86.8 \%$ female; age $74.78 \pm 5.72$ years, height $1.56 \pm 0.08$ $\mathrm{m}$, weight $67.13 \pm 12.14 \mathrm{~kg}$ ), from Baixo Alentejo, Portugal. The study involved collecting indicators on the participants' sociodemographic characteristics, the history of falls, fear of fall (FES-I Portugal scale); level of physical activity (IPAQ-E - short version) and physical fitness indicators (Rikli \& Jones, 2013). There were significant associations between fear of falling and physical fitness variables. Low fear of falling seems to be related to better lower strength $(p<.001)$, agility and dynamic balance $(p<.001)$, and cardiorespiratory fitness $(p$ $<.001$ ). reporting a relationship of increased fear of falling and decreased physical fitness levels. The results reveal that a low fear of falling is related to better functional capacity. In order to contribute to the maintenance and development of essential physical capacities and avoid a decrease in quality of life, we reinforce the importance of physical exercise programs that include strength, agility, balance and cardiorespiratory fitness, integrated in multifactorial interventions. These strategies can, through the promotion and maintenance of functional capacity, create a better perception of competence and consequently reduce the fear of falling in older adults.

Keywords: Strength, Healthy aging, Falls, Exercise, Health Promotion.

Resumen. El miedo a caer se señala como una consecuencia psicológica resultante de la pérdida de funcionalidad y equilibrio inherente al envejecimiento. Mantener buenos niveles de aptitud física puede ser la clave para preservar la calidad de vida y la independencia de los mayores. Este estudio tiene como objetivo verificar la relación del miedo a caer con la aptitud física de mayores inscriptos en programas de ejercicio físico. La muestra incluyó 308 mayores, con edad ${ }^{3} 65$ años (86.8\% mujeres; edad $74.78 \pm 5.72$ años, altura 1.56 $\pm 0.08 \mathrm{~m}$, peso $67.13 \pm 12.14 \mathrm{~kg}$ ), del BaixoAlentejo, Portugal. Se evaluarán características sociodemográficas, historial de caídas, miedo a caer (FES-I Portugal), nivel de actividad física (IPAQ-E - versión corta) y indicadores de capacidad física (Rikli \& Jones, 2013). Se observaron relaciones significativas entre el miedo a caer y las variables de aptitud física. Bajo medo a caer parece estar relacionado con una mejor fuerza de los miembros inferiores $(p<.001)$, agilidad y equilibrio dinámico $(\mathrm{p}<.001)$ y aptitud cardiorrespiratoria $(p<$ .001). Los resultados revelan que un bajo medo a caer está relacionado con una mejor capacidad funcional. Con el fin de contribuir al mantenimiento y desarrollo de la aptitud física y evitar una disminución de la calidad de vida, reforzamos la importancia de los programas de ejercicio físico que incluyen fuerza, agilidad, equilibrio y aptitud cardiorrespiratoria, integrados en intervenciones multifactoriales. Estas estrategias pueden, a través de la promoción y el mantenimiento de las capacidades funcionales, crear una mayor percepción de competencia y, en consecuencia, reducir el miedo a caer.

Palabras clave: Fuerza, Envejecimiento saludable, Caídas, Ejercicio, Promoción de la salud.

Fecha recepción: 26-03-21. Fecha de aceptación: 18-07-21

Vania Azevedo Ferreira Brandao de Loureiro

vloureiro@ipbeja.pt 


\section{Introdução}

Portugal é um dos países da União Europeia com maior taxa de inatividade física, em que mais de $60 \%$ dos adultos não pratica desporto ou atividade física $\mathrm{e}$ adultos acima dos 65 anos apresentam maiores índices de inatividade física (Direção-Geral da Saúde, 2017). Portugal é um dos países da União Europeia com maior taxa de inatividade física, em que mais de $60 \%$ dos adultos não pratica desporto ou atividade física e adultos acima dos 65 anos apresentam maiores índices de inatividade física (Direção-Geral da Saúde, 2017). Aliado a esse facto está a evidência de que na última década se tem denotado um aumento considerável no envelhecimento na população da união europeia (Corselli-Nordblad \& Strandell, 2020). Portugal (21,8\%) surge como o terceiro país, tal como a Finlândia, com a percentagem mais elevada de pessoas com 65 anos ou mais, na população total; a primeira e segunda percentagem pertencem à Itália $(22,8 \%)$ e à Grécia (22.0\%) (Corselli-Nordblad \& Strandell, 2020).

Dadas as adversidades subjacentes à inatividade física refletidas na saúde e ao panorama demográfico, surge como necessidade prioritária modificar esse fator comportamental (Direção-Geral da Saúde, 2017). Como parte integrante da solução, o exercício físico tem sido proposto como possível intervenção não farmacológica (André, Ferrand, Albinet, \& Audiffren, 2018). Recentes revisões sistemáticas sustentam ainda que a prática regular de exercício tem efeitos anti envelhecimento e multi sistémicos (Silva, AldoradinCabeza, Eslick, Phu, \& Duque, 2017) na medida em que reduz o risco de baixa capacidade funcional, baixos níveis de aptidão física, declínio cognitivo e fragilidade (Apóstolo et al., 2018; Chase, Phillips, \& Brown, 2017; Silva et al., 2017; Tejada Medina, Díaz Caro, González García, \& Ruiz Montero, 2020). A este respeito, importa expor a evidência de que os programas de exercício poderão ser eficazes na prevenção de quedas em 23\% (Barreto, Rolland,Vellas, \& Maltais, 2019; Gomes, Manzano, \& Loureiro, 2019).

A ocorrência de quedas advém de uma natureza multifatorial (Gillespie et al., 2012) dependente de fatores intrínsecos, como aspetos fisiológicos, músculoesqueléticos e psicossociais associados ao envelhecimento, e extrínsecos, relacionados com o ambiente (Loureiro, Paixão, \& Castillo-Viera, 2021). O impacto que os fatores extrínsecos têm no risco de quedas ainda é incerto (Todd \& Skelton, 2004). Em casa, incluem riscos como pisos escorregadios, escadas, iluminação insuficientes, tapetes mais soltos, calçado e vestuário, e em locais públicos incluem mau design de edifícios, piso escorregadio, calçadas rachadas ou irregulares e uma má iluminação (WHO, 2008). As quedas são um problema entre adultos com 65 ou mais anos (Verma et al., 2016) com resultados adversos para a saúde, tais como lesões (Gazibara et al., 2017; Verma et al., 2016), hospitalização (Manemann et al., 2016) e morte (Manemann et al., 2016; WHO, 2008).

O medo de cair, a inatividade física e a restrição das atividades da vida diária são fatores preeminentes considerados como causas comportamentais associadas a episódios de queda (Jin, 2018; Lusardi et al., 2017). O medo de cair afeta muitos idosos, independentemente de terem realmente experimentado quedas (Jefferis et al., 2014). Além de constituir um forte fator preditivo de quedas (Gazibara et al., 2017), o medo de cair é também apontado como consequência psicológica proveniente da perda de funcionalidade e equilíbrio inerentes ao envelhecimento (Delbaere et al., 2010) e está associado a resultados negativos para a saúde física e psicossocial, incluindo a depressão (Mishra, Mishra, \& Bidija, 2017) e a restrição de atividade (Bruce, Hunter, Peters, Davis, \& Davis, 2015). As quedas e, em particular, o medo de cair são relevantes barreiras que impedem que os idosos obtenham benefícios para a saúde através da caminhada e da atividade física moderada a vigorosa (Jefferis et al., 2014).

Nos últimos anos, a literatura tem identificado o treino de equilíbrio como uma das estratégias mais eficazes na promoção do envelhecimento ativo, na medida em que reduz o índice de risco de queda (Sherrington et al., 2020), assim como o constrangimento psicológico do medo de cair (Garber et al., 2011; Loureiro et al., 2021). Desenvolver habilidades que permitam à população idosa manter bons níveis de aptidão física é a chave para manter a sua qualidade de vida e independência (Rikli \& Jones, 2013). Neste sentido, sabe-se que a condição física inclui componentes de força muscular, flexibilidade, velocidade motora, capacidade aeróbia, equilíbrio dinâmico, é definida como a capacidade fisiológica de realizar atividades adequadas, com segurança e independência sem fadiga excessiva (Gschwind et al., 2013). Contudo, garantir manutenção da capacidade de equilíbrio no desempenho de tarefas da via diária e na preservação de independência, bem como na reação ativa a perturbações externas do equilíbrio (Treacy et al., 2015) contempla um desafio complexo.

Tendo em consideração os factos anteriormente 
expostos, o presente estudo teve como objetivo verificar qual a relação do medo de cair com a aptidão física de idosos inseridos em programas de exercício físico (EF) municipais e a viver na comunidade. Baseado em literatura relevante (Loureiro et al., 2019), a hipótese estabelecida no presente estudo é que o medo de cair poderá influenciar negativamente os níveis de aptidão física da população idosa.

\section{Material e Métodos}

O objetivo do presente estudo foi verificar qual a relação do medo de cair com a aptidão física de idosos inseridos em programas de exercício físico (EF) municipais e a viver na comunidade. Os programas de EF municipais estão alinhados com as diretrizes do projeto UP Again Senior. O projeto, cujos objetivos centrais visam consciencializar a população idosa para a importância da atividade física na saúde e fomentar a autonomia funcional e qualidade de vida através de programas de exercício físico, organiza as sessões de exercício de acordo com o modelo FITT: frequência de 2 sessões/semana; intensidade moderada; duração de 45 minutos; tipo de atividade aeróbica, resistência muscular e flexibilidade (Riebe et al., 2018). Os programas de EF municipais são ministrados por profissionais de exercício físico especializados.

\section{Participantes}

O estudo observacional descritivo incorporou uma amostra de conveniência, contemplando 308 idosos, com idades 65 anos (86.8\% do sexo feminino; idade média $74.78 \pm 5.72$ anos, altura $1.56 \pm 0.08 \mathrm{~m}$, peso $67.13 \pm$ $12.14 \mathrm{~kg}$ ), pertencentes à região do Baixo Alentejo, distrito de Beja, Portugal. Os participantes no programa de exercício, após aceitação, foram convocados via telefone para proceder às avaliações nos diferentes locais com maior proximidade das respetivas residências. As avaliações foram implementadas por técnicos de EF qualificados. Os critérios de inclusão no presente estudo foram: participantes com idade $>65$ anos, condição de saúde estável e/ou controlada; capacidade de compreender e executar instruções; marcha independente sem utilização de auxílio na marcha (ex.: andarilhos, canadianas); não apresentarem contraindicação médica para a prática de EF. Os critérios de condição de saúde estável e/ou controlada e de ausência de contraindicação médica para a prática de EF foram verificados no momento de inscrição no programa de EF através da apresentação de atestado médico.
O atestado médico foi solicitado, por cada um dos participantes, ao médico de família, no centro de saúde local.

Os participantes foram informados sobre os procedimentos de avaliação, como também acerca dos seus riscos e benefícios. Um consentimento informado por escrito foi assinado pelos participantes. A participação foi voluntária, sendo garantido o anonimato e confidencialidade de cada participante. O estudo foi aprovado pela Comissão de Ética do Instituto Politécnico de Beja (CEIPBeja n. $\left.{ }^{\circ}: 03 / 2019\right)$. O estudo seguiu os padrões éticos da Declaração de Helsínquia.

\section{Procedimentos e Instrumentos}

A recolha de dados foi estruturada em três momentos sequenciais de avaliação, realizados no mesmo dia e decorreu no período de março a maio de 2019. No primeiro momento, os participantes procederam ao autopreenchimento de um questionário, que permitiu a recolha de indicadores relativos às suas características sociodemográficas (grupo etário, género, estado civil, local de residência e toma de medicamentos), bem como do histórico de quedas («Teve alguma queda nos últimos 12 meses?»). No segundo momento foram autoavaliados o nível de atividade física e o medo de cair. No terceiro momento os técnicos de EF avaliaram os indicadores de saúde e aptidão física funcional. Todas as avaliações foram realizadas por uma equipa de investigadores especializados, treinados para o efeito.

Nível de atividade física. O IPAQ, versão curta, é recomendado para estudos alargados da população pois é mais fácil, mais rápida e viável para preencher (Bauman et al., 2009). A determinação do nível de atividade física foi obtida com recurso ao Questionário Internacional de Atividade Física (IPAQ-E) - versão curta. O IPAQ E, destinado a adultos com 65 ou mais anos, está disponível em www.ipaq.ki.se (International Physical Activity Questionnaire, 2009), e apenas se encontra validado para o Reino Unido (Cleland, Ferguson, Ellis, \& Hunter, 2018) e Suécia (Hurtig-Wennlöf, Hagströmer, \& Olsson, 2010).

Medo de Cair. O medo de cair foi determinado através da: Falls Efficacy Scale International - FES-I (Delbaere et al., 2010). Esta escala encontra-se adaptada e validada para a população portuguesa (MarquesVieira, Sousa, Sousa, \& Berenguer, 2018) e apresenta uma elevada fiabilidade (Kempen et al., 2008). Avalia o medo de cair ao realizar 16 atividades distintas e cada item é pontuado de 1 (nada preocupado) a 4 (muito preocupado). O medo de cair foi classificado como baixo 
(16-19 pontos), moderado (20-27 pontos) ou elevado (28-64 pontos) (Refacho, Santos, Magalhães, Lourenço, \& Fernandes, 2019).

Medidas Antropométricas. Todas as medidas antropométricas foram avaliadas segundo o padrão internacional (Marfell-Jones, Stewart, \& Ridder, 2012), sendo obtidas antes de qualquer teste físico. A massa corporal (em kg) foi medida através de balança padrão (Tanita SC-330, Reino Unido) com aproximação de 0.1 kg. Para a estatura (em m), foi utilizado estadiómetro de precisão com escala de $0.001 \mathrm{~m}$ (Seca, modelo 213, Alemanha). O índice de massa corporal (IMC) foi obtido a partir da equação (Anjos, 1992) que utiliza o peso (em $\mathrm{kg}$ ) dividido pela altura (m) ao quadrado, isto é, IMC = peso / altura ${ }^{2}$. A estratificação do IMC foi realizada segundo os parâmetros recomendados pela Direção-Geral da Saúde (Direção-Geral da Saúde, 2013).

Aptidão Física Funcional. Os testes de avaliação da aptidão física funcional foram realizados segundo a bateria de testes de Rikli e Jones (2013). Esta bateria, composta por 7 testes, avalia a força de membros inferiores (MI) (teste levantar e sentar); força de membros superiores (MS) (teste de flexão do antebraço); flexibilidade de flexibilidade de MI (teste sentar e alcançar); flexibilidade de MS (teste de alcançar atrás das costas); agilidade e equilíbrio dinâmico (teste sentado, caminhar 2,44 m e voltar a sentar), composição corporal (teste da estatura e peso), e aptidão cardiorrespiratória (teste andar 6 minutos) (Baptista \& Sardinha, 2005).

\section{Análise Estatística}

Para caracterização total da amostra recorreu-se à apresentação da média $(\bar{x})$ e desvio padrão (DP) para variáveis contínuas e para variáveis categóricas, a frequência (n) e percentagem (\%). A homogeneidade das variáveis foi testada através do teste de Levene. Para cada umas das variáveis dependentes (isto é, histórico de quedas, equilíbrio dinâmico e força muscular) foi aplicada a análise de variância One Way ANOVA, com teste post hoc de Tukey, para comparação dos grupos: «baixo medo de cair», «moderado medo de cair» e «elevado medo de cair». Para verificar a correlação entre as diferentes variáveis qualitativas, recorremos ao coeficiente de correlação de Pearson. A análise estatística foi

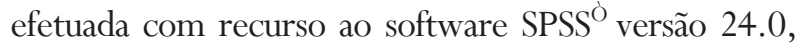
tendo-se fixado um nível de significância $p<.05$.

\section{Resultados}

A análise dos resultados revela que a maioria dos participantes, da região de Beja, são do sexo feminino (86.8\%), vive acompanhado/a (63.6\%) e apresenta como habilitações literárias o $1^{\circ}$ ciclo de escolaridade (68.8\%). Como indicadores de risco de queda foi possível verificar que a prescrição de fármacos está presente quase na totalidade dos participantes (96.4\%). Reportamos ainda que, considerando o IMC, apenas $24.4 \%$ dos participantes apresenta valores normais e $75.0 \%$ apresentam excesso de peso e/ou obesidade. A queda, nos últimos 12 meses, foi reportada por $46.2 \%$ dos participantes, apresentando uma correlação baixa, mas significativa com o nível de preocupação em cair durante a realização de AVD's $(r=0.226 ; p<.001)$. Relativamente ao nível de AF, 40.0\% dos idosos realiza AF vigorosa. Contudo, e apesar de serem idosos integrados em programas de EF, ainda se verifica que $35.1 \%$ reporta baixos níveis de AF. A caracterização da amostra, em função da classificação de medo de cair, é apresentada na Tabela 1.

\begin{tabular}{|c|c|c|c|c|c|c|}
\hline & & $\begin{array}{c}\text { Baixo medo de } \\
\text { cair } \mathrm{n}(\%)\end{array}$ & $\begin{array}{l}\text { Moderado medo de } \\
\quad \text { cair } \mathrm{n}(\%)\end{array}$ & $\begin{array}{c}\text { Elevado medo de } \\
\text { cair n }(\%)\end{array}$ & Total & $P$ \\
\hline \multirow[b]{2}{*}{ Idade $^{-}$(DP) } & & $96(31.2)$ & $108(35.1)$ & $104(33.8)$ & 308 & \multirow[b]{2}{*}{.053} \\
\hline & Anos & $73.9 \pm 5.8$ & $74.5 \pm 5.5$ & $75.8 \pm 5.8$ & $74.8 \pm 5.7$ & \\
\hline \multirow{2}{*}{ Género n (\%) } & Feminino & $75(78.1)$ & $97(89.8)$ & $95(91.3)$ & $267(86.8)$ & \multirow{2}{*}{$.011 *$} \\
\hline & Masculino & $21(21.9)$ & $11(10.2)$ & $9(8.7)$ & $41(13.3)$ & \\
\hline \multirow{6}{*}{ Escolaridade n (\%) } & $1^{\circ}$ ciclo EB & $68(70.8)$ & $75(69.4)$ & $69(66.3)$ & $212(68.8)$ & \multirow{6}{*}{$.008 *$} \\
\hline & $2^{\circ}$ ciclo EB & $5(5.2)$ & $9(8.3)$ & $6(5.8)$ & $20(6.5)$ & \\
\hline & $3^{\circ}$ ciclo EB & $9(9.4)$ & $5(4.6)$ & $5(4.8)$ & $19(6.2)$ & \\
\hline & Ensino Secundário & $5(5.2)$ & $9(8.3)$ & $2(1.9)$ & $16(5.2)$ & \\
\hline & Ensino Superior & $3(3.1)$ & $3(2.8)$ & $0(0)$ & $6(1.9)$ & \\
\hline & Analfabeto & $6(6.3)$ & $7(6.5)$ & $22(21.2)$ & $35(11.4)$ & \\
\hline \multirow{3}{*}{$\begin{array}{c}\text { Com quem vive } \mathrm{n} \\
(\%)\end{array}$} & Família & $68(70.8)$ & $72(66.7)$ & $56(53.8)$ & $196(63.6)$ & \multirow{3}{*}{.079} \\
\hline & Sozinho & $28(9.1)$ & $36(33.3)$ & $47(45.2)$ & $111(36.0)$ & \\
\hline & Lar & $0(0.0)$ & $0(0.0)$ & $1(0.3)$ & $1(0.4)$ & \\
\hline \multirow{2}{*}{ Medicação n (\%) } & Sim & $92(95.8)$ & $105(97.2)$ & $100(96.2)$ & $297(96.4)$ & \multirow{2}{*}{.853} \\
\hline & Não & $4(4.2)$ & $3(2.8)$ & $4(3.8)$ & $11(3.6)$ & \\
\hline \multirow{3}{*}{ Nível de AF n (\%) } & Leve & $43(44.8)$ & $37(34.3)$ & $28(26.9)$ & $108(35.1)$ & \multirow{3}{*}{$.002 *$} \\
\hline & Moderada & $16(16.7)$ & $21(19.4)$ & $39(37.5)$ & $76(24.7)$ & \\
\hline & Vigorosa & $37(38.5)$ & $50(46.3)$ & $37(35.6)$ & $124(40.3)$ & \\
\hline \multirow{6}{*}{$\operatorname{IMC} n(\%)$} & Peso reduzido & $0(0.0)$ & $0(0.0)$ & $2(0.6)$ & $2(0.6)$ & \multirow{6}{*}{.075} \\
\hline & Peso normal & $32(10.4)$ & $21(6.8)$ & $22(7.1)$ & $75(24.4)$ & \\
\hline & Excesso de peso & $43(14)$ & $58(18.8)$ & $43(14)$ & $144(46.8)$ & \\
\hline & Obesidade Grau I & $18(5.8)$ & $21(6.8)$ & $27(8.8)$ & $66(21.4)$ & \\
\hline & Obesidade Grau II & $2(0.6)$ & $8(2.6)$ & $9(2.9)$ & $19(6.2)$ & \\
\hline & Obesidade Grau III & $1(0.3)$ & $0(0.0)$ & $1(0.3)$ & $2(0.6)$ & \\
\hline Queda nos últimos & Não & $77(80.2)$ & $73(67.6)$ & $56(53.8)$ & $56(53.8)$ & \multirow{2}{*}{$<.001 * *$} \\
\hline 12 meses n (\%) & Sim & $19(19.8)$ & $35(32.4)$ & $48(46.2)$ & $48(46.2)$ & \\
\hline
\end{tabular}

Relativamente ao medo de cair verificou-se que $68.9 \%$ dos idosos revelou preocupação moderada (35.1\%) e elevada em cair (33.8\%).

Foram identificadas associações significativas entre o medo de cair e as variáveis de aptidão física $(p<$ .005). O baixo medo de cair está associado a melhores resultados nas variáveis de força muscular dos MI (F $\left.{ }_{(2,305)}=13.945 ; p<.001\right)$, agilidade e equilíbrio dinâmico $\left(\mathrm{F}_{(2,305)}=12.449 ; p<.001\right)$ e aptidão cardiorrespiratória $\left(\mathrm{F}_{(2,305)}=11.345 ; p<.001\right)$. O medo de cair e a força dos MS, flexibilidade dos MI e dos MS não revelaram 
associações significativas $(p>.05)$. Os resultados da relação entre o medo de cair e as variáveis de aptidão física são apresentados na tabela 2.

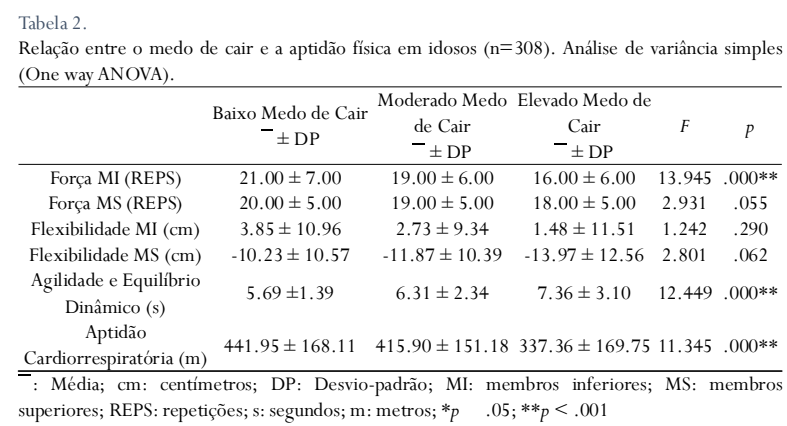

\section{Discussão}

O presente estudo procurou verificar qual a relação do medo de cair com a aptidão física de idosos inseridos em programas de EF municipais e a viver na comunidade. $\mathrm{Na}$ literatura há poucos estudos com o intuito de pesquisar a utilização de exercícios para intervir no medo de cair em idosos. Em linha com estudos prévios, o medo de cair é caracterizado como fator de risco intrínseco, de saúde mental, em episódios de quedas e determinante de funcionalidade e qualidade de vida na população idosa (Lusardi et al., 2017) . Os resultados do presente estudo revelam que o baixo medo de cair está associado com melhores índices de aptidão física, em particular na força dos MI (21.00 \pm 7.00 reps $)$, agilidade e equilíbrio dinâmico $(5.69 \pm 1.39 \mathrm{~s})$ e aptidão cardiorrespiratória $(441.95 \pm 168.11 \mathrm{~m})$. A relação entre o medo de cair e a restrição de realização de atividades está documentada na literatura (Lenze \& Wetherell, 2011) e, o medo de cair à luz da sua associação com a condição física, condiciona a participação em atividades físicas e funcionais por medo da incapacidade em realizá-las (Chua, Jiang, Lim, Wu, \& Wang, 2019). Apesar da atividade física ser um fator-chave para a manutenção da saúde, incluindo a competência funcional (Chodzko-Zajko et al., 2009; Martínez Heredia, Santaella Rodríguez, \& Rodríguez-García, 2020), o desempenho de qualquer tarefa física, incluindo atividades diárias, está associado a um certo risco de queda e por essa razão, muitos idosos residentes na comunidade podem acreditar que a inatividade é a melhor forma de eliminar ou reduzir o risco (Wijlhuizen, Chorus, \& Hopman-Rock, 2010). Considerando o exposto, a prática de EF regular pode, numa perspetiva biopsicossocial, ser considerada benéfica (Bull et al., 2020; Pedersen \& Saltin, 2015). Em Portugal, as consultas de aconselhamento em atividade física no
Serviço de Saúde são estratégias recentes na qual se pretende promover a importância da prática de atividade física em todas as fases do ciclo de vida (Madeira, Sousa, Envia, Marques, \& Moreira, 2018; P. Silva, Graça, Mata, Arriaga, \& Silva, 2016). Adotar um novo comportamento ativo poderá ser uma importante estratégia para melhorar a compreensão do comportamento relacionado à AF. Através da mudança comportamental, futuramente sugere-se que programas municipais consolidem programas de AF para a comunidade, organizados com avaliações do estado de saúde e capacidade funcional periódicas, intervenções regulares de EF (em grupo ou sessões de treino em casa) e sessões de literacia em saúde (Loureiro et al., 2021; Madeira et al., 2018). De facto, as intervenções multifatoriais, com integração de equipas multidisciplinares, têm vindo a revelar-se como estratégias eficientes na manutenção e promoção das capacidades funcionais, bem como na diminuição do índice de quedas face ao medo de cair em pessoas idosas residentes na comunidade (Gomes et al., 2019; Hopewell et al., 2018). O estudo apresenta algumas limitações, o medo de cair foi determinado através de questionário, não sendo considerados a grande variedade de fatores já conhecidos que estão associados ao medo de cair, concretamente a idade mais avançada, o sexo feminino, quedas anteriores, declínio cognitivo, fragilidade, ansiedade, tonturas, má auto-avaliação da saúde, depressão, problemas de marcha, baixos recursos económicos e viver sozinho (Kumar, Carpenter, Morris, Iliffe, \& Kendrick, 2014). Outras limitações estão relacionadas com as características da amostra de estudo, maioritariamente feminina, baixo nível de escolaridade, do contexto específico do Baixo Alentejo e com histórico de prática de exercício físico, o que pode ter implicações na interpretação dos dados.

\section{Conclusão}

O medo de cair parece associar-se às variáveis de aptidão física e na população estudada um baixo medo de cair está relacionado com melhores indicadores de força dos MI, agilidade e equilíbrio dinâmico e de aptidão cardiorrespiratória. No sentido de contribuir para a manutenção e desenvolvimento de capacidades físicas essenciais e evitar uma diminuição de qualidade de vida ou independência, poderemos assumir a importância de programas multicomponente de EF que incluam o treino de força, agilidade, equilíbrio e aptidão cardiorrespiratória, integrados em intervenções 
multifatoriais. Estas estratégias poderão, através da promoção e manutenção das capacidades funcionais dos idosos residentes na comunidade, criar uma maior perceção de competência e consequentemente reduzir o medo de cair.

Para efetivas medidas de promoção de saúde, qualidade de vida e de prevenção de quedas, são necessários mais estudos que investiguem os modelos de previsão, com recurso ao histórico de queda, medo de cair, velocidade de marcha, toma e tipo de medicação, problemas visuais, entre outros, de modo a prever quedas. $\mathrm{O}$ atual envelhecimento populacional fomenta a necessidade de se investigarem, numa perspetiva biopsicossocial e ecológica, os aspetos fundamentais para a personalização das intervenções destinadas a idosos, quer para a resolução do medo de cair, para a manutenção e promoção das capacidades funcionais, bem como para adoção de comportamentos ativos e saudáveis, onde se inclui a prática de atividade física.

\section{Agradecimentos}

As autoras gostariam de agradecer aos participantes da presente investigação, às Câmaras Municipais do Distrito de Beja e aos técnicos de exercício físico pela sua colaboração. O presente estudo foi apoiado pelo Instituto Português da Juventude, através do Programa Nacional Desporto para Todos 2020 (PNDpT - IPDJ, CP/532/DDT/2020).

\section{Referências}

André, N., Ferrand, C., Albinet, C., \& Audiffren, M. (2018). Cognitive Strategies and Physical Activity in Older Adults: A Discriminant Analysis. Journal of aging research, 2018. doi:https://doi.org/10.1155/ 2018/8917535

Apóstolo, J., Cooke, R., Bobrowicz-Campos, E., Santana, S., Marcucci, M., Cano, A., . . . Holland, C. (2018). Effectiveness of interventions to prevent prefrailty and frailty progression in older adults: a systematic review. JBI database of systematic reviews and implementation reports, 16(1), 140-232. doi:https:// doi.org/10.11124/JBISRIR-2017-003382

Baptista, F., \& Sardinha, L. (2005). Avaliação da Aptidão Física e do Equilíbrio de Pessoas Idosas - Baterias de Fullerton. Lisboa: Faculdade de Motricidade Humana,.

Barreto, P. d. S., Rolland, Y., Vellas, B., \& Maltais, M.
(2019). Association of Long-term Exercise Training With Risk of Falls, Fractures, Hospitalizations, and Mortality in Older Adults: A Systematic Review and Meta-analysis. JAMA internal medicine, 179(3), 394405 .doi:https:// doi.org/10.1001/ jamainternmed.2018.5406

Bauman, A., Bull, F., Chey, T., C., C., Ainsworth, B., Sallis, J., . . . The IPS Group. (2009).The International Prevalence Study on Physical Activity: results from 20 countries. International Journal of Behavioral Nutrition and Physical Activity, 6(21). doi:https: / / doi.org/10.1186/1479-5868-6-21

Bruce, D., Hunter, M., Peters, K., Davis, T., \& Davis, W. (2015). Fear of falling is common in patients with type 2 diabetes and is associated with increased risk of falls. Age Ageing, 44, 687-690. doi:10.1093/ageing/ afv024

Bull, F. C., Al-Ansari, S. S., Biddle, S., Borodulin, K., Buman, M. P., Cardon, G., ... Willumsen, J. F. (2020). World Health Organization 2020 guidelines on physical activity and sedentary behaviour. British Journal of Sports Medicine, 54(24). doi:10.1136/ bjsports-2020-102955

Chase, J. A. D., Phillips, L. J., \& Brown, M. (2017). Physical Activity Intervention Effects on Physical Function Among Community-Dwelling Older Adults: A Systematic Review and Meta-Analysis. Journal of Aging and Physical Activity, 25(1), 149-170. doi:https://doi.org/10.1123/japa.2016-0040

Chodzko-Zajko, W. J., Proctor, D. N., Fiatarone Singh, M. A., Minson, C. T., Nigg, C. R., Salem, G. J., \& Skinner, J. S. (2009). American College of Sports Medicine position stand. Exercise and physical activity for older adults. Med Sci Sports Exerc, 41(7), 1510-1530. doi:10.1249/MSS.0b013e3181a0c95c

Chua, C. H. M., Jiang, Y., Lim, D. S., Wu, V. X., \& Wang,W. (2019). Effectiveness of cognitive behaviour therapy-based multicomponent interventions on fear of falling among community-dwelling older adults: A systematic review and meta-analysis. Journal of Advanced Nursing, 75(12), 3299-3315. doi:https: / / doi.org/10.1111/jan.14150

Cleland, C., Ferguson, S., Ellis, G., \& Hunter, R. F. (2018). Validity of the International Physical Activity Questionnaire (IPAQ) for assessing moderate-tovigorous physical activity and sedentary behaviour of older adults in the United Kingdom. BMC medical research methodology, 18(1). doi:https://doi.org/ 10.1186/s12874-018-0642-3

Corselli-Nordblad, L., \& Strandell, H. (2020). Ageing 
Europe: Looking at the Lives of Older People in the EU. In EUROSTAT (Ed.). doi:10.2785/628105

Delbaere, K., Close, J., Mikolaizak, A., Sachdev, P., Brodaty, H., \& Lord, S. (2010). The Falls Efficacy Scale International (FES-I). A comprehensive longitudinal validation study. Age and Ageing, 39, 210 216. doi: https: / / doi.org/10.1093/ageing/afp225

Direção-Geral da Saúde. (2017). Programa Nacional para a Promoção da Atividade Física. Retrieved from https: / /www.sns.gov.pt/wp-content/uploads/2017/10/ DGS_PNPAF2017_V7.pdf

Garber, C. E., Blissmer, B., Deschenes, M. R., Franklin, B. A., Lamonte, M. J., Lee, I.-M., . . . Swain, D. P. (2011). Quantity and Quality of Exercise for Developing and Maintaining Cardiorespiratory, Musculoskeletal, and Neuromotor Fitness in Apparently Healthy Adults. Medicine \& Science in Sports \& Exercise, 43(7), 1334-1359. doi:ttps://doi.org/ 10.1249/MSS.0b013e318213fefb

Gazibara, T., Kurtagic, I., Kisic-Tepavcevic, D., Nurkovic, S., Kovacevic, N., Gazibara, T., \& Pekmezovic, T. (2017). Falls, risk factors and fear of falling among persons older than 65 years of age. Psychogeriatrics, 17, 215-223. doi:10.1111/psyg. 12217

Gillespie, L. D., Robertson, M. C., Gillespie, W. J., Sherrington, C., Gates, S., Clemson, L. M., \& Lamb, S. E. (2012). Interventions for preventing falls in older people living in the community. The Cochrane Database of Systematic Reviews, 9(9), 1-416. doi:https: / /doi.org/10.1002/14651858.CD007146.pub3

Gomes, M., Manzano, S., \& Loureiro, V. (2019). The consequence of exercise interventions on rate of falls and balance in Multifactorial Interventions: Systematic Review. Paper presented at the International Congress of Health and Well-being Intervention.

Hopewell, S., Adedire, O., Copsey, B., Boniface, G., Sherrington, C., Clemson, L., . . Lamb, S. (2018). Multifactorial and multiple component interventions for preventing falls in older people living in the community (Review ). Cochrane Database of Systematic Reviews(7). doi:https://doi.org/10.1002/ 14651858.CD012221.pub2.www.cochranelibrary.com Hurtig-Wennlöf, A., Hagströmer, M., \& Olsson, L. (2010). The International Physical Activity Questionnaire modified for the elderly: Aspects of validity and feasibility. Public Health Nutrition, 13(11), 1847-1854. doi:10.1017/S1368980010000157

International Physical Activity Questionnaire. (2009). International Physical Activity Questionnaire (2009) International Physical Activity Questionnaire downloads. Retrieved from https:// sites.google.com/site/theipaq/questionnaire_links Jefferis, B. J., Iliffe, S., Kendrick, D., Kerse, N., Trost, S., Lennon, L. T., . . Whincup, P. H. (2014). How are falls and fear of falling associated with objectively measured physical activity in a cohort of communitydwelling older men? BMC Geriatrics, 14(1), 114. doi:https: / /doi.org/10.1186/1471-2318-14-114

Jin, J. (2018). Prevention of Falls in Older Adults. JAMA, 319(16). doi:doi:10.1001/jama.2018.4396

Kempen, G., Yardley, L., Haastregt, J., Zijlstra, G., Beyer, N., Hauer, K., \& Todd, C. (2008). The Short FES-I: a shortened version of the falls efficacy scaleinternational to assess fear of falling. Age Ageing, 37(1), 45-50. doi:https://doi.org/10.1093/ageing/afm157

Kumar, A., Carpenter, H., Morris, R., Iliffe, S., \& Kendrick, D. (2014). Which factors are associated with fear of falling in community-dwelling older people? Age and Ageing, 43(1), 76-84. doi:https:// doi.org/10.1093/ageing/aft154

Lenze, E. J., \& Wetherell, J. L. (2011). A lifespan view of anxiety disorders. Dialogues in Clinical Neurosciences, 13, 381-399.

Loureiro, V., Paixão, C., \& Castillo-Viera, E. (2021). Prevención de Caídas y Ejercício Físico como Determinantes de la Salud de las Personas Mayores. In M. Mendoza-Sierra \& E. Moreno-Sanchez (Eds.), La longevidad como transformación social en el siglo XXI (pp. 65-84): Editiones ALJIBE.

Lusardi, M., Fritz, S., Middleton, A., Allison, L., Wingood, M., Phillips, E., . . . Chui, K. (2017). Determining Risk of falls in community dwelling older adults: A systematic review and meta-analysis using posttest probability. Journal of Geriatric Physical Therapy, 40(1). doi:https://doi.org/10.1519/ JPT.000000000000009

Madeira, S. G., Sousa, P. A., Envia, G., Marques, S., \& Moreira, T. M. (2018). Physical activity counseling: A proposed clinical intervention flowchart. Acta Medica Portuguesa, 31(6), 295-298. doi:https:// doi.org/10.20344/amp.9920

Manemann, S. M., Chamberlain, A. M., Boyd, C., Weston, S. A., Jiang, R., \& Roger, V. L. (2016). Fall risk and outcomes in cardiovascular disease-A community study. Circulation, 134(A15665).

Marfell-Jones, M. J., Stewart, A. D., \& Ridder, J. H. (2012). International standards for anthropometric assessment - ISAK: International Society for the Advancement of Kinanthropometry,

Marques-Vieira, C. M. A., Sousa, L. M. M., Sousa, L. 
M. R., \& Berenguer, S. M. A. C. (2018). Validation of the Falls Efficacy Scale - International in a sample of Portuguese elderly. Revista Brasileira de Enfermagem, 71, 747-754. doi:https://doi.org/10.1590/00347167-2017-0497

Martínez Heredia, N., Santaella Rodríguez, E., \& Rodríguez-García, A.-M. (2020). Beneficios de la actividad física para la promoción de un envejecimiento activo en personas mayores. Revisión bibliográfica. Retos, 39, 829-834. doi:https://doi.org/ $10.47197 /$ retos.v0i39.74537

Mishra, N., Mishra, A. K., \& Bidija, M. (2017). A study quality of life in elderly individuals. International Journal of Research In Medical Sciences, 5, 1456-1460. doi:10.18203/2320-6012.ijrms20171245

Pedersen, B. K., \& Saltin, B. (2015). Exercise as medicine - Evidence for prescribing exercise as therapy in 26 different chronic diseases. Scandinavian Journal of Medicine and Science in Sports, 24, 1-72. doi: https: / /doi.org/10.1111/sms.12581

Refacho, A., Santos, J., Magalhães, M., Lourenço, T., \& Fernandes, B. (2019). Efeitos do treino orientado para a tarefa na marcha, equilíbrio e medo de cair após acidente vascular cerebral: estudo de caso. Saúde \&Tecnologia ÚDE \&TECNOLOGIA . NOVEMBRO | 2019 | \#22 | P. 28-33 .ISSN: , 28-33. Validation of Criterion-Referenced Clinically Relevant Fitness Standards for Maintaining Physical Independence in LaterYears. The Gerontologist, 53(2), 255-267. doi:https://doi.org/10.1093/geront/ gns071

Sherrington, C., Fairhall, N., Kwok, W., Wallbank, G., Tiedemann, A., Michaleff,Z., . . . Bauman,A. (2020). Evidence on physical activity and falls prevention for people aged $65+$ years: systematic review to inform the WHO guidelines on physical activity and sedentary behaviour. International Journal of Behavioral on correlation between depression, fear of fall and

Rikli, R. E., \& Jones, C. J. (2013). Development and

Nutrition and Physical Activity, Int J Behav Nutr Phys Act 17, 144(2020). (144). doi:https://doi.org/10.1186/ s12966-020-01041-3

Silva, P., Graça, P., Mata, F., Arriaga, M. T., \& Silva, A. J. (2016). Estratégia Nacional para a Promoção da Atividade Física, da Saúde e do Bem-Estar. Lisboa: DGS Retrieved from https://www.dgs.pt/documentos-epublicacoes/estrategia-nacional-para-a-promocaoda-atividade-fisica-da-saude-e-do-bem-estar-pdf.aspx Silva, R., Aldoradin-Cabeza, H., Eslick, G. D., Phu, S., \& Duque, G. (2017). The effect of physical exercise on frail older persons: a systematic review. The Journal of Frailty \& Aging, 6(2), 91-96. doi:https: / / doi.org/ $10.14283 /$ jfa. 2017.7

Tejada Medina, V., Díaz Caro, C., González García, C., \& Ruiz Montero, P. (2020). Programas de intervención física en mujeres mayores a través del método Pilates: Una revisión sistemática. Retos, 39, 10061016. doi:https://doi.org/10.47197/ retos.v0i39.78005

Todd, C., \& Skelton, D. (2004). What are the main risk factors for falls among older people and what are the most effective interventions to prevent these falls? Retrieved from http://www.euro.who.int/document/ E82552.pdf,

Verma , S. K., Willetts, J. L., Corns, H. L., MarucciWellman, H. R., Lombardi, D. A., \& Courtney, T. K. (2016). Falls and fall-related injuries among community-dwelling adults in the United States. PLOS ONE, 11. doi:10.1371/journal.pone.0150939.

WHO. (2008). WHO global report on falls prevention in older age. Retrieved from https: / / apps.who.int/iris / handle/10665/43811

Wijhuizen, G. J., Chorus, A. M., \& Hopman-Rock, M. (2010). The FARE: a new way to express FAlls Risk among older persons including physical activity as a measure of exposure. Preventive medicine, 50(3), 143147. doi:https://doi.org/10.1016/ j.ypmed.2009.12.014
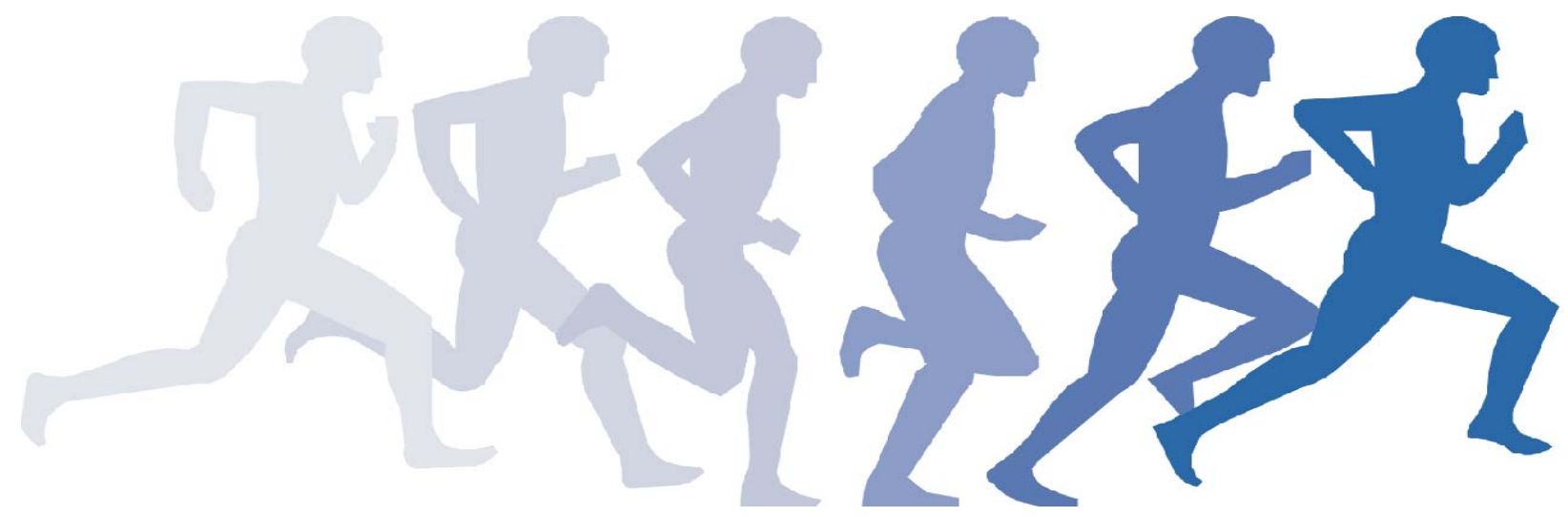